\title{
The role of high resolution computed tomography in the diagnosis of interstitial lung disease
}

\author{
David M Hansell, Ian H Kerr
}

Until recently the chest radiograph has been the only imaging technique used in the assessment of patients with suspected diffuse lung disease. In this context the chest radiograph is less than ideal. Problems arise with both false negative and false positive results. It is well established that the chest radiograph may appear entirely normal in up to $10 \%$ of patients with biopsy proved diffuse lung disease of various causes, ${ }^{1}$ and a poor quality chest radiograph, especially of an obese patient, may misleadingly raise the spectre of diffuse lung disease. The two dimensional nature of a chest radiograph dictates that there is superimposition of structures over the lungs and it has been estimated that up to $40 \%$ of the lungs is obscured in normal subjects. This obscuration will be further compounded by the presence of pleural thickening or an effusion. Even when the chest radiograph shows definite evidence of diffuse lung disease McCloud has pointed out that "the chest radiograph is often non-specific. Various radiographic patterns, together with their predominant location, correlate statistically with their pathologic entities but in individual cases the chest roentgenogram is rarely diagnostic."'2

Until recently the main use for computed tomography has been detection of pulmonary nodules and, despite some pioneering work by Kreel in the early $1980 \mathrm{~s}^{3}{ }^{3}$ it has had a limited role in the investigation of diffuse lung disease. Improvements in computed tomography scanner technology, notably in terms of spatial resolution and shorter scan times, have led to renewed interest in the application of computed tomography to show the fine morphological detail of the lung. Research in this area is particularly active and is reflected in the large volume of descriptive work on the computed tomographic appearances of many diffuse lung diseases that has appeared over the last five years ${ }^{4}$; there are no signs that this period of research is over. The technique of high resolution computed tomography, used to show the lung parenchyma, ${ }^{5}$ is sufficiently different from conventional computed tomography to warrant a brief description.

Conventional computed tomography of the thorax uses contiguous $1 \mathrm{~cm}$ sections; in this way the entire lungs are included in one study. This protocol is widely used when a comprehensive examination of the lungs is required-for example, in the search for metastases. The volume averaging that occurs within the $1 \mathrm{~cm}$ thickness of the scan, however, substantially reduces the ability of conventional computed tomography to resolve small structures. For high resolution computed tomography the section thickness is reduced to $1-3 \mathrm{~mm}$ and a different software reconstruction of the image is used to improve spatial resolution (figs $1 a$ and $1 b$ ). These scans are interspaced by at least $1 \mathrm{~cm}$. Where $3 \mathrm{~mm}$ sections are taken $1 \mathrm{~cm}$ apart the radiation dose to the breast is reduced to about half that of conventional computed tomography. None the less, the radiation burden inherent in high resolution computed tomography is considerable and in a complete study using $3 \mathrm{~mm}$ sections every $1 \mathrm{~cm}$ the dose is roughly 40 times that received from a single chest radiograph. $^{6}$

Because high resolution computed tomography includes only very short segments of pulmonary vessels these narrow sections may be misinterpreted as showing a nodular pattern. For this reason some radiologists advocate conventional computed tomography before high resolution computed tomography but this obviously adds to the radiation dose. Because, by definition, diffuse lung disease is widespread many radiologists now perform a limited number of high resolution computed tomography scans, perhaps as few as six, in such patients. In this way not only is the radiation dose restricted but the cost of the examination is not much more than that of a posteroanterior and lateral chest radiograph. It has been estimated in the United States that high resolution computed tomography used in this way costs $\$ 180$, compared with $\$ 90$ for a posteroanterior and lateral chest radiograph. In the same comparison a transbronchial biopsy with bronchoalveolar lavage costs $\$ 1500$ and an open lung biopsy $\$ 5000$ (R A Webb, personal communication). Computed tomography is becoming more widely available throughout the United Kingdom and most computed tomography machines installed over the last three years are able to provide high resolution images.

The spatial resolution of the latest gener- 
Figure 1 (a) Conventional $10 \mathrm{~mm}$ width computed tomographic section through the upper lobe normal). (b) High resolution $3 \mathrm{~mm}$ width computed tomographic section at the same level. Note the different appearance of the blood iessels and bronchi.

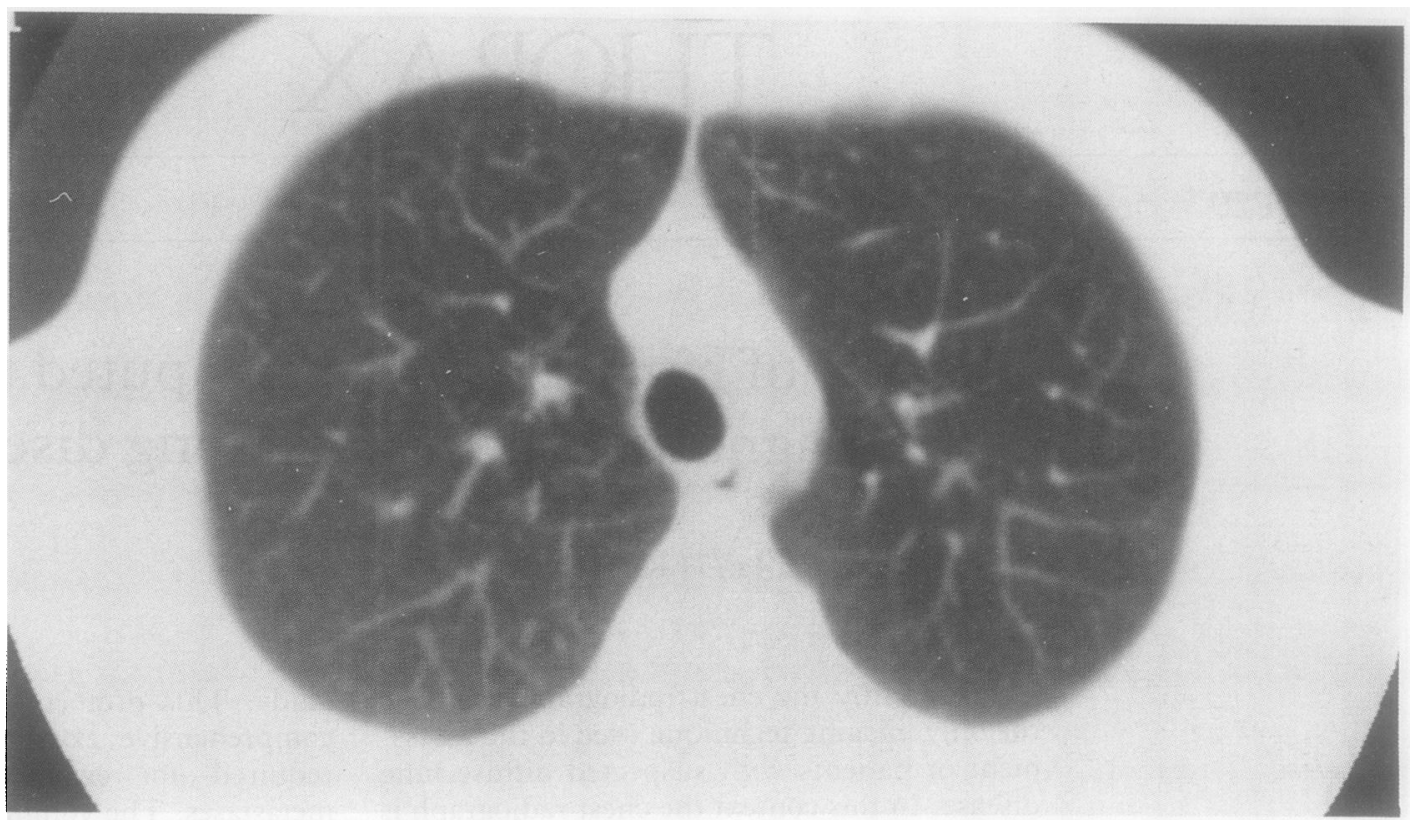

(a)

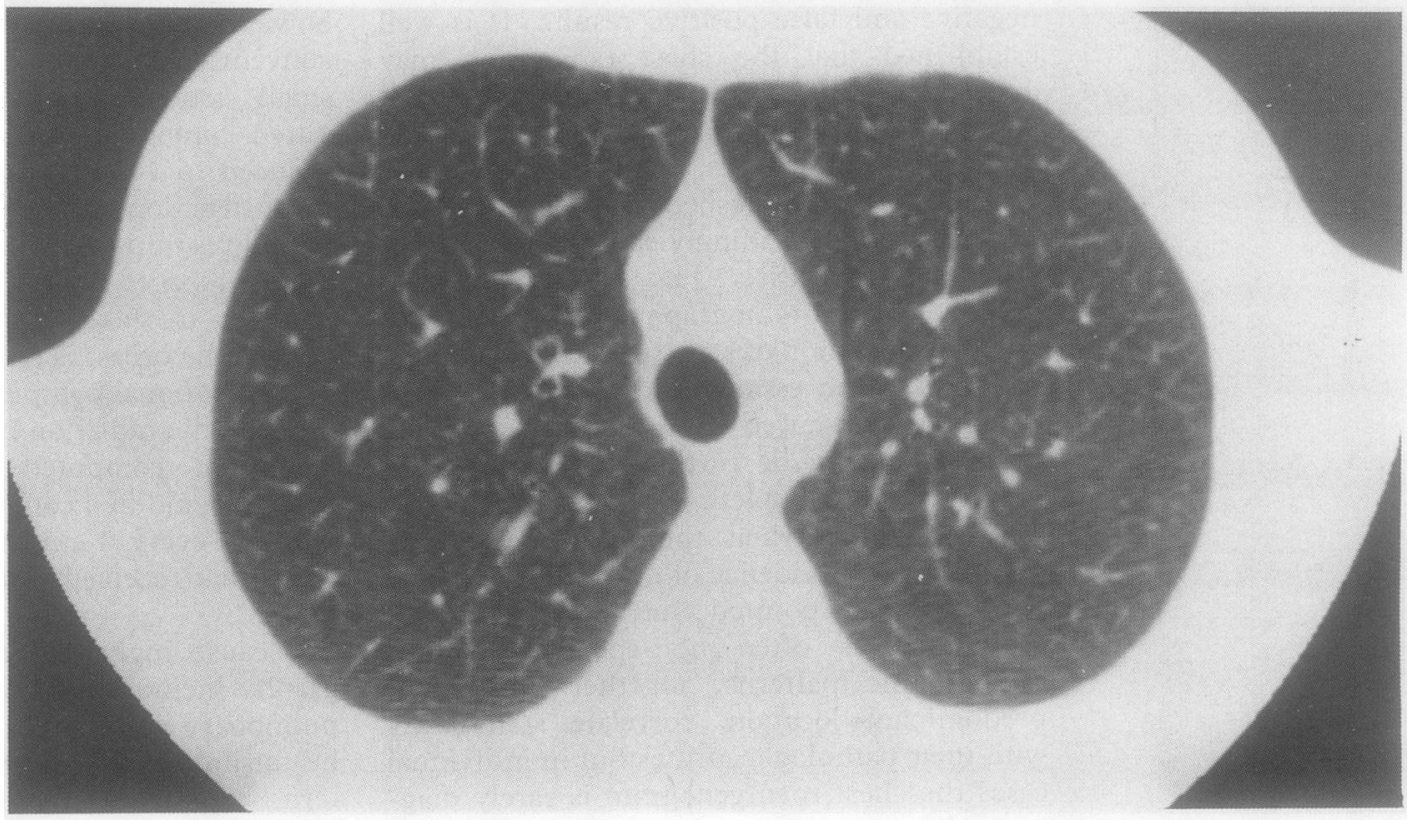

$(b)$

ation of computed tomography scanners cnables structures as small as $200 \mu \mathrm{m}$ to be resolved. ${ }^{i}$ As a result the outline of the basic unit of the lung, the secondary pulmonary lobule, can often be identified. The interlobular septum measures $100 \mu \mathrm{m}$ across in normal individuals and is therefore more often identified in pathological states when there is thickening of the interstitium (fig 2). These septa, analogous to the Kerley B septal lines seen in a different plane on the chest radiograph, are seen most frequently in the periphery of the anterolateral parts of the lung and over the surface of the diaphragm. The secondary pulmonary lobule itself is an irregular polyhedron and contains a connective tissue sheath that surrounds the bronchovascular bundle. This core may be visible as a small dot in the centre of the lobule (fig 3).

Attempts to discriminate so called "airspace" from "interstitial" disease by inferences from the pattern on a chest radiograph have largely been discredited. ${ }^{49}$ This is partly because the abnormal shadowing on a radiograph results from summation of numerous foci; the chest radiograph has been fancifully described as "the two-dimensional projectional sum of several thousand histologic slices." "1" Another compelling reason is the overwhelming frequency with which interstitial and airspace lesions coexist in any given disease (see fig 2). Nevertheless, high resolution computed tomography does show interstitial thickening precisely in the few diseases in which the pathological process is largely confined to the interstitium. An example is shown in figure 4; the chest radiograph of this patient showed non-specific shadowing in the right upper zone. Despite extensive investigation a diagnosis was not reached. High resolution computed tomography through the upper lobes shows unilateral thickening of the bronchovascular bundles and interlobular septa, features 
Figure 2 a) Polygonal outlines representing thickened interlobular septa (arrowheads) in silicosis. The background nodularity is more obvious on sections through the upper zones. (b) Thickening of the interlobular septa in areas of airspace consolidation due to alveolar proteinosis.

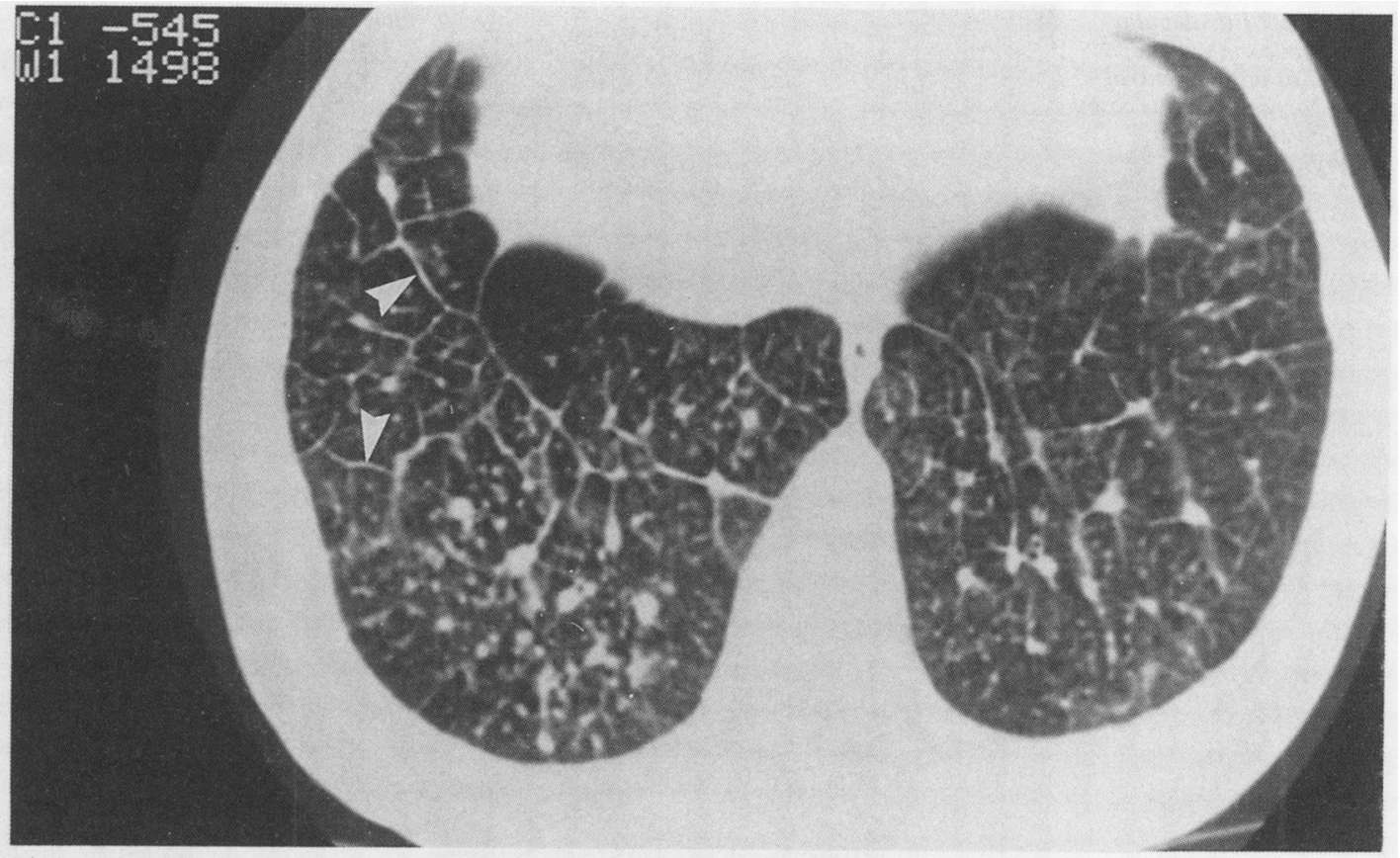

(a)

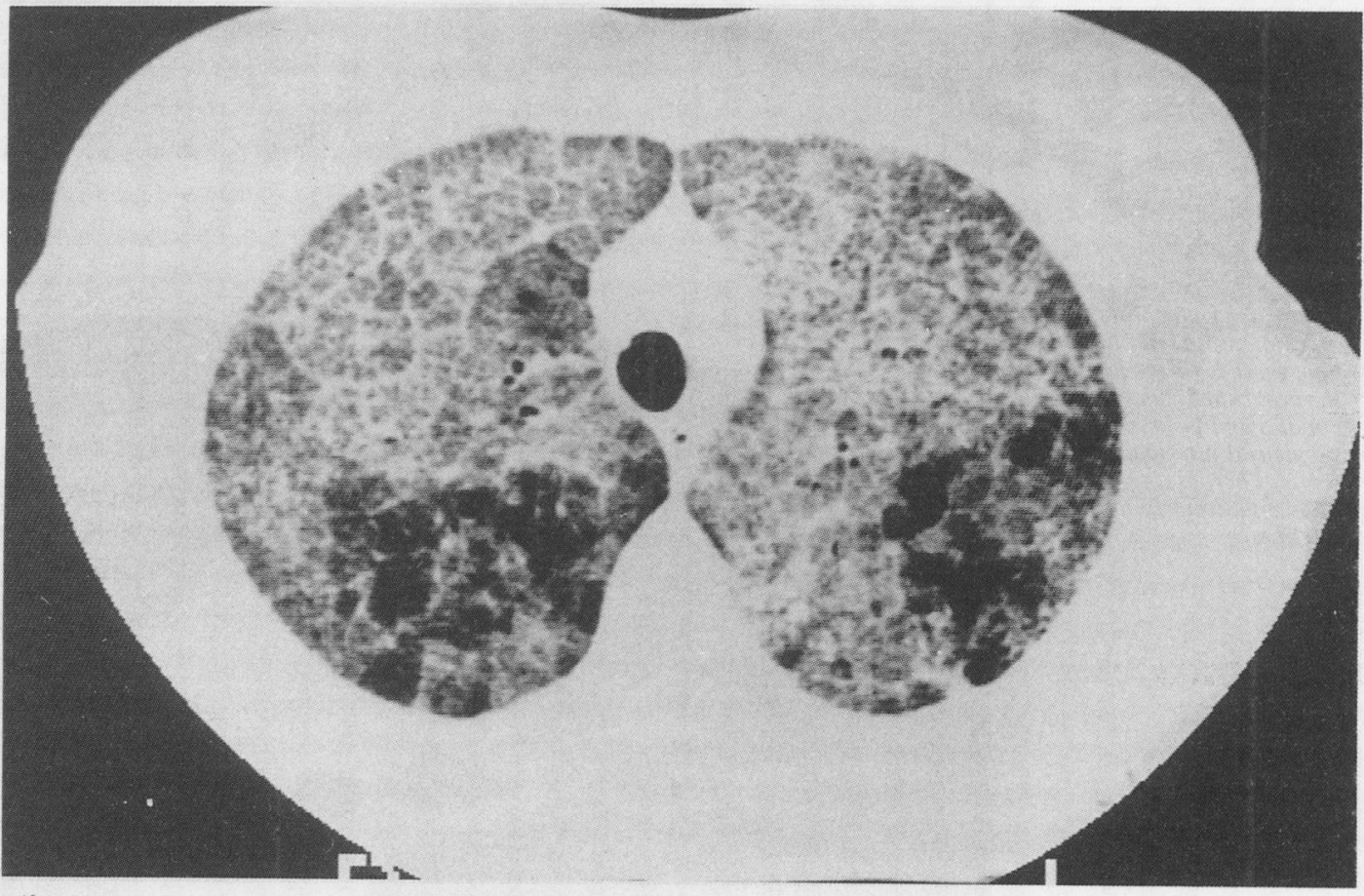

$(b$

characteristic of lymphangitis carcinomatosa. ${ }^{11}$ From the pathological descriptions of lymphangitis the high resolution computed tomography appearances can be predicted and the same applies to pulmonary sarcoidosis. ${ }^{1}$ There are subtle but definite features that differentiate the high resolution computed tomography appearances of sarcidosis from lymphangitis carcinomatosa: in sarcoidosis the nodular thickening of the bronchovascular bundles is more pronounced and thickening of the interlobular septa less obvious (fig 5). Nevertheless, as with chest radiography, pulmonary sarcoidosis has many guises and a wide range of high resolution computed tomography appearances has been reported ${ }^{13}$ (fig 6). Fibrosing alveolitis, whether "lone" or associated with other diseases, has been shown to have a characteristic computed tomographic appear- ance. The crescentic subpleural distribution concentrated in the posterobasal segments of the lower lobes is virtually pathognomonic ${ }^{1415}$ (fig 7). Not surprisingly, given this basal distribution, high resolution computed tomoradiograph. ${ }^{16}$ Scans taken in the prone position may be helpful in distinguishing parenchymal opacification due to increased perfusion of the dependent parts of the lung from minimal fibrosing alveolitis. As the fibrosis progresses a widespread, coarse reticular pattern supervenes, resulting in a non-specific appearance of end stage pulmonary fibrosis. In the clinical context of unexplained deterioration of a patient with fibrosing alveolitis high resolution computed tomography may be of value as it may reveal other disease-for example, a bronchogenic carcinoma. High resolution comgraphy will show disease despite a normal chest 
Figure 3 Intralobular bronchovascular bundles visible as minute central dots (arrows) in this case of subacute extrinsic allergic alveolitis.

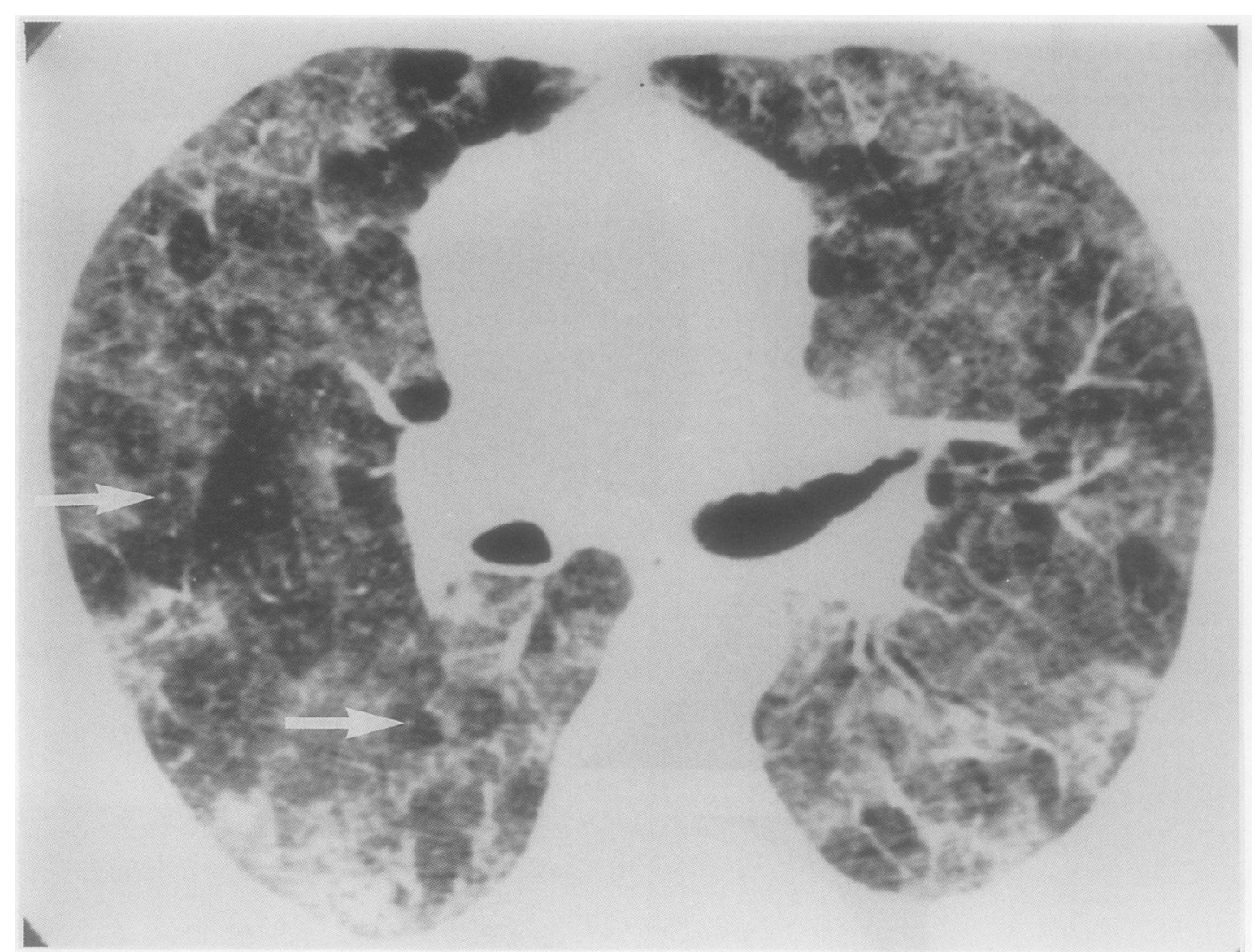

Figure 4 Lymphangitis carcinomatosa: in the righ lung there is thickening of the bronchovascular bundles and prominent subpleural interlobular septa. There is a pleural plaque posteriorly in the left chest.

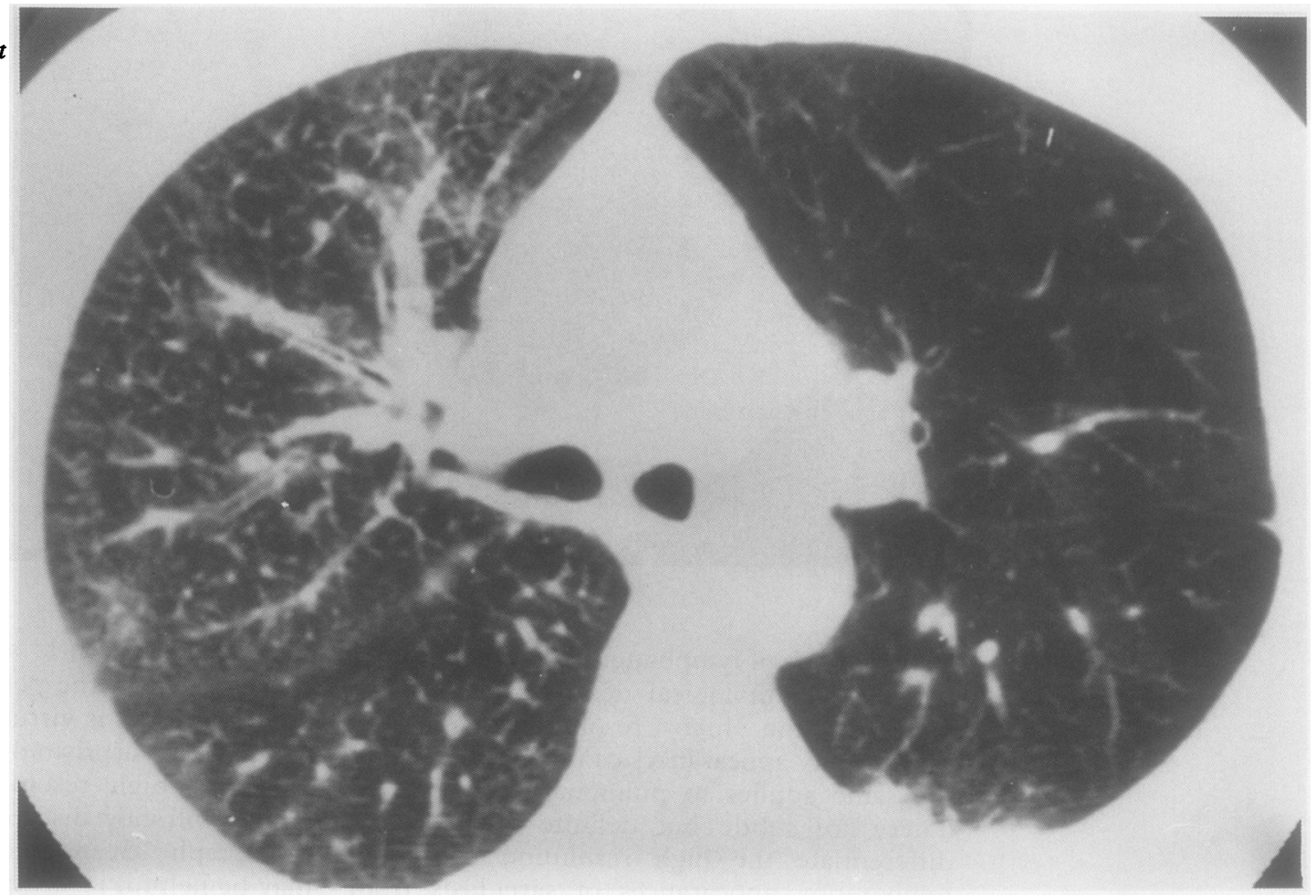

puted tomography may also show coexisting emphysema, ${ }^{17}$ which may be impossible to detect by chest radiography. There is increasing evidence of a high degree of correlation between high resolution computed tomography appearances and the amount of disease activity in fibrosing alveolitis in terms of the proportion of cellular infiltrate to established fibrosis found histologically. The observation that amorphous parenchymal opacification represents increased cellularity, in both the airspaces and the interstitium (fig 8), whereas the reticular pattern is the result of fibrosis (fig 7) appears, with some provisos, to be true. ${ }^{18}{ }^{19}$ No other diagnostic technique (including lung function tests $^{20}$ ) short of biopsy allows this 
Figure 5 Sarcoidosis, with nodules

predominantly

concentrated around

bronchovascular bundles.

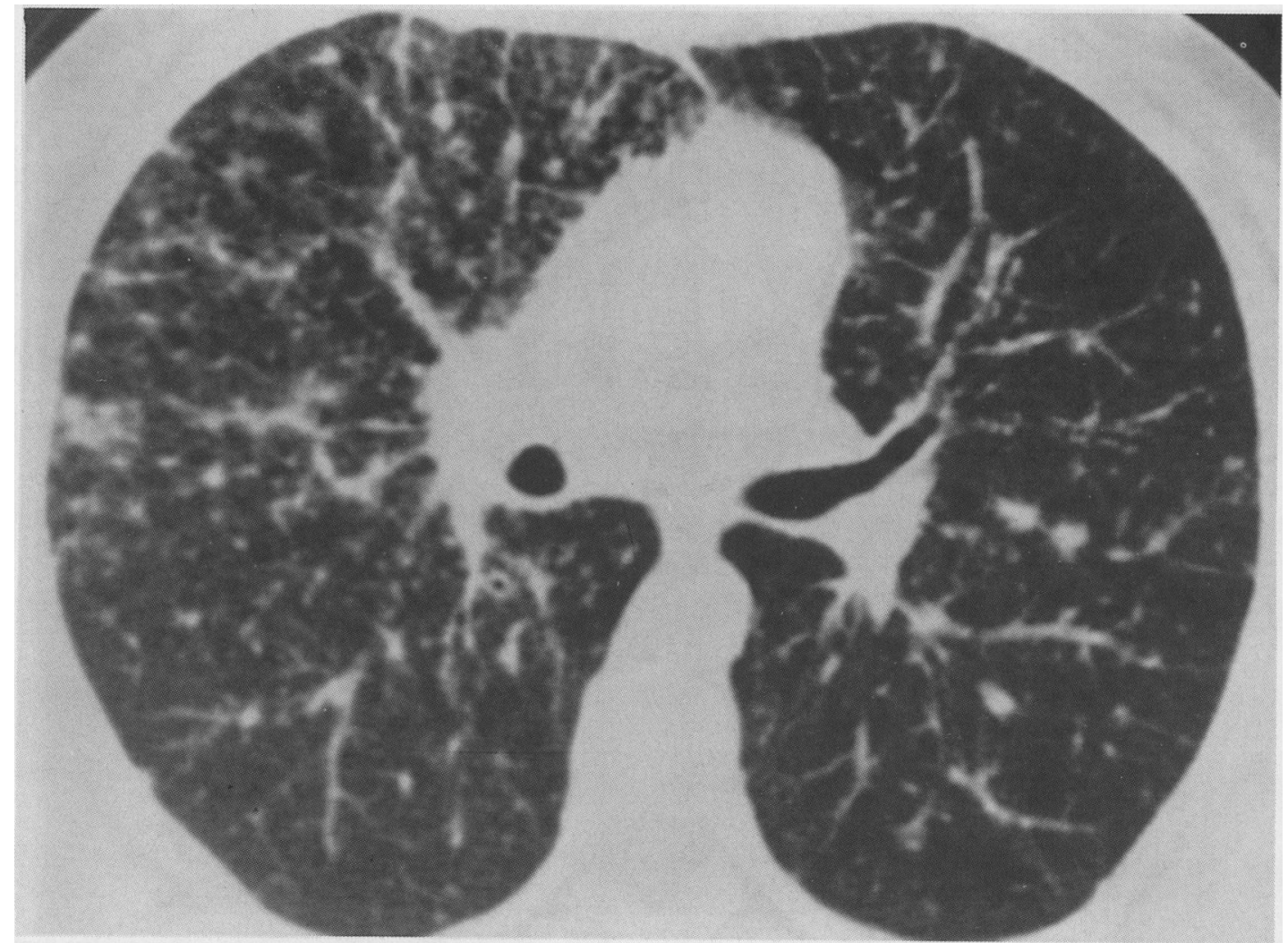

Figure 6 Sarcoidosis: a less common pattern with irregular areas of confluent parenchymal opacification (prone scan).

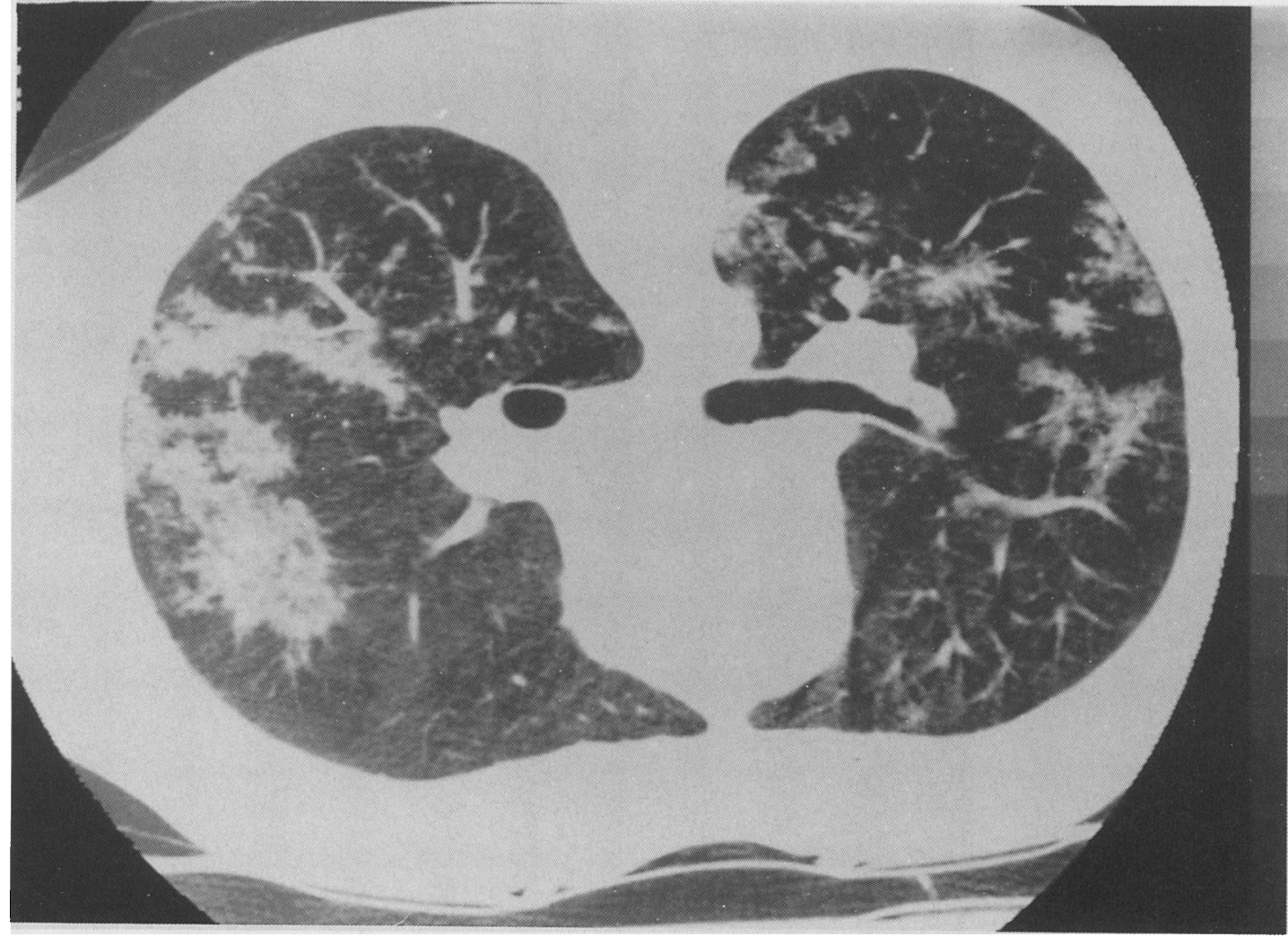

distinction to be made reliably.

The superior sensitivity of high resolution computed tomography for detecting asbestosis is now undisputed, particularly in those patients with extensive pleural disease, which obscures the underlying lung. ${ }^{21}{ }^{22}$ High resolution computed tomography, however, has opened a window on minor degrees of pleuroparenchymal abnormality whose long term significance is as yet unknown. It will be some years before the natural history of these changes is established. Other conditions in which high resolution computed tomography frequently shows parenchymal abnormality in 
Figure 7 Cryptogenic fibrosing alveolitis: the typical subpleural distribution of the reticular pattern of established fibrosis (prone scan).

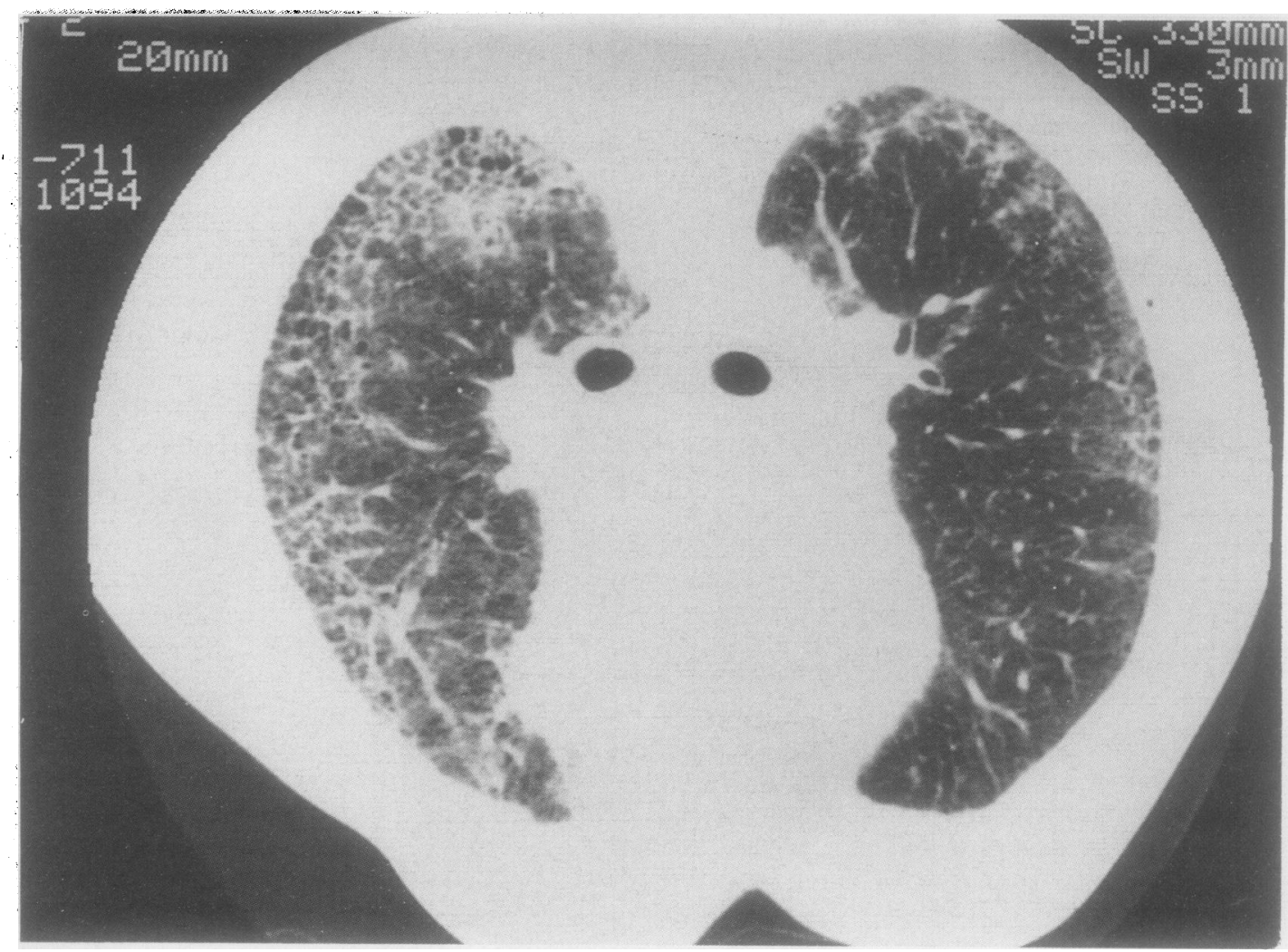

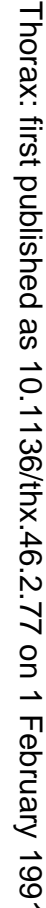

Figure 8 Crytogenic fibrosing alveolitis: poorly defined parenchymal opacification corresponding to $a$ predominantly cellular infiltration in the interstitium and airspaces (prone scan).

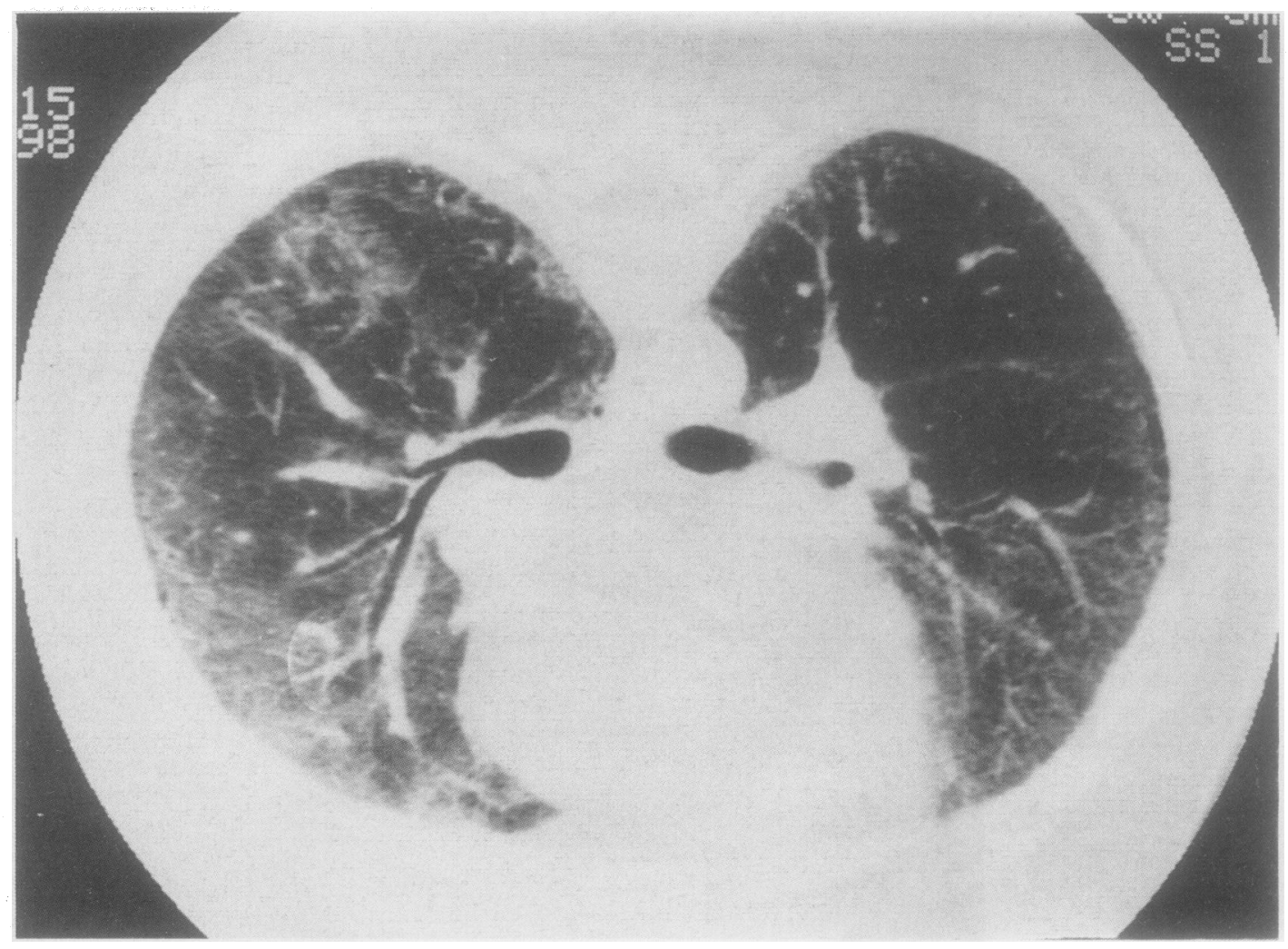

the face of a normal chest radiograph include subacute extrinsic allergic alveolitis ${ }^{23}$ (fig 9) and drug induced lung damage. ${ }^{24}$ The high resolution computed tomography patterns of most of the rarer diffuse lung diseases have now been described in detail. These include the pulmonary manifestations of lymphangioleiomyomatosis, ${ }^{2526}$ histiocytosis $\mathbf{X},{ }^{27}$ and tuberous sclerosis. ${ }^{28}$ Although these patterns may not in themselves be unerringly diagnostic, in a given clinical context they will often confirm the diagnosis and at the same time exclude other suspected diseases, such as emphysema. The example of lymphangioleiomyomatosis shown in figure 10 demonstrates numerous cystic air spaces of uniform 
Figure 9 Subacute extrinsic allergic alveolitis: global parenchymal opacification due to infiltration of the interstitium and airspaces. $A$ concurrent chest radiograph was normal.

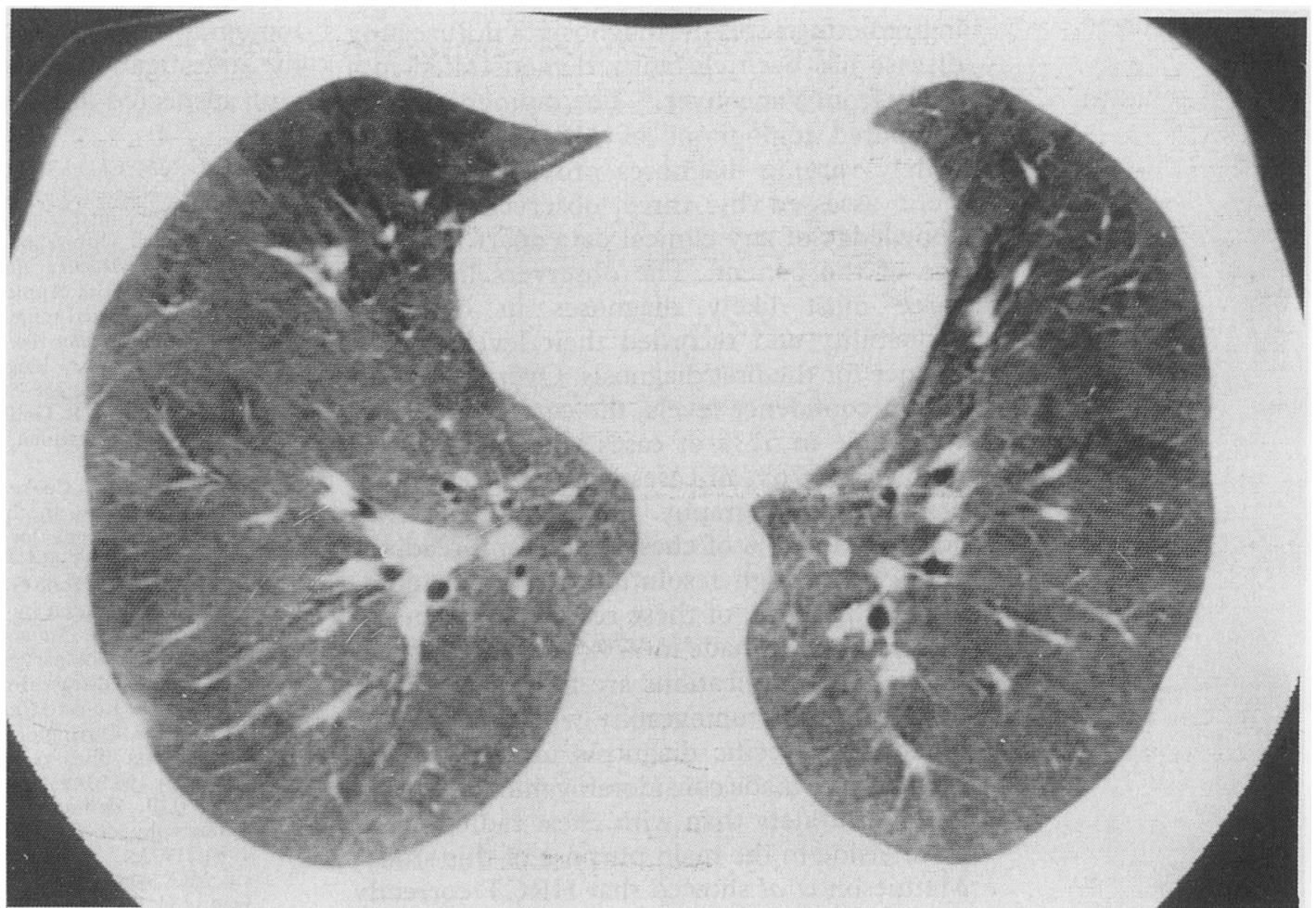

\section{Figure 10}

Lymphangioleiomyomatosis: well defined cystic airspaces. Note the preservation of a normal vascular pattern. The chest radiograph showed minimal and non-specific changes.

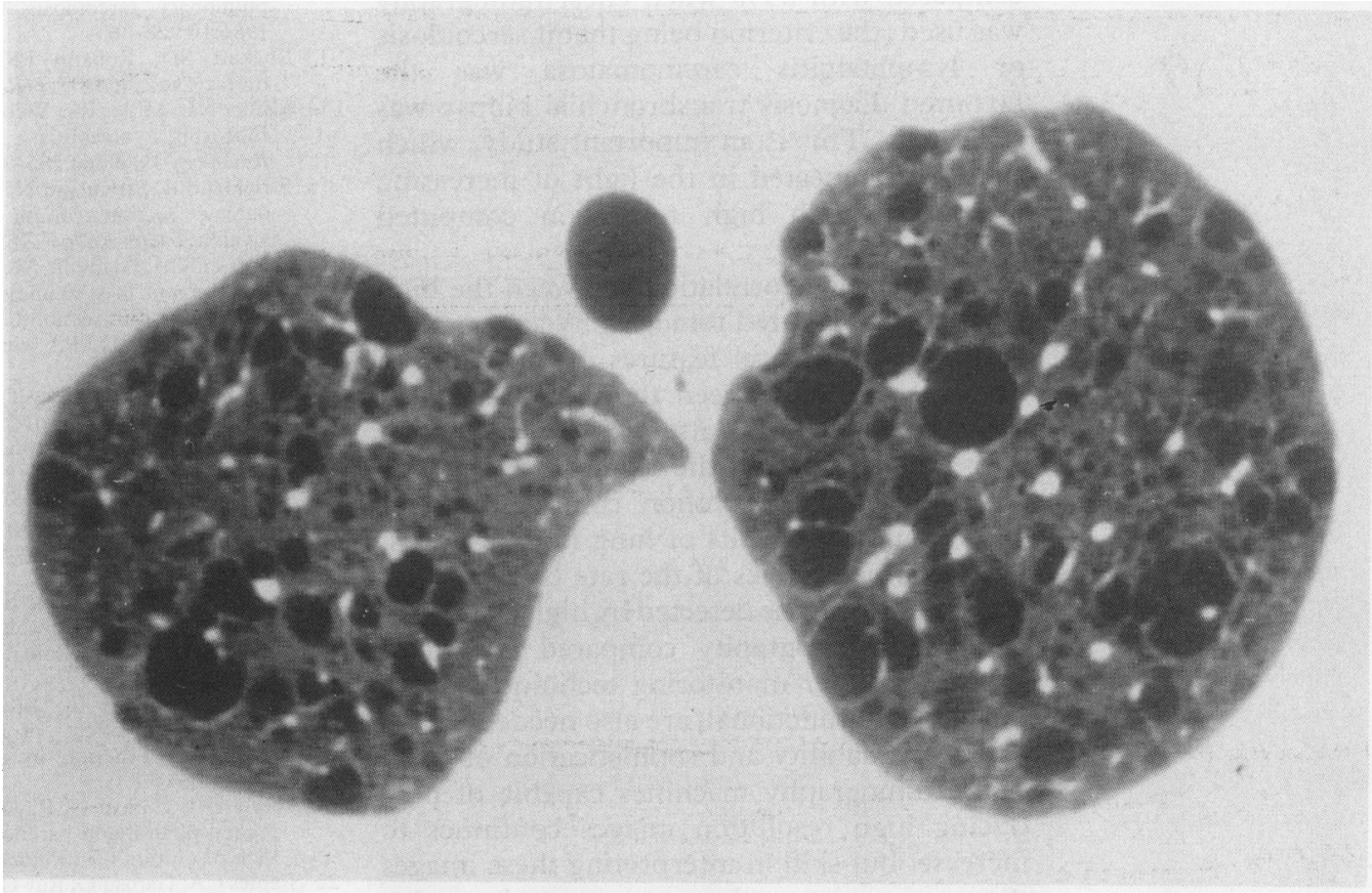

size with fine but definable walls (in contradistinction to the areas of lung destruction seen in emphysema, which do not have discernible margins). This woman with lymphangioleiomyomatosis had a spontaneous pneumothorax during pregnancy; the underlying lungs appeared questionably abnormal on the chest radiograph. In this instance a definite diagnosis was made from the high resolution computed tomography appearances without resorting to an open lung biopsy.

Although the sensitivity of high resolution computed tomography in detecting minor degrees of diffuse lung disease is not seriously challenged by any other imaging technique currently available, its precise role in the dyspnoeic patient with a normal chest radiograph and abnormal results in lung function tests remains to be determined. It seems likely that high resolution computed tomography will obviate the need for lung biopsy by providing a specific diagnosis in some of these patients. In patients needing a biopsy high used to provide guidance to the site most likely to yield satisfactory histological material.

The utility of high resolution computed tomography compared with chest radiography resolution computed tomography should be 
in predicting a specific diagnosis of diffuse lung disease has been elegantly demonstrated in a study from Vancouver. ${ }^{29}$ The radiographs and computed tomograms of 118 patients, with widely varying diagnoses proved by biopsy, were assessed by three observers without knowledge of any clinical data apart from the age of the patient. The observers listed the three most likely diagnoses in order of probability and recorded their level of confidence for the first diagnosis. Overall, regardless of confidence levels, the correct diagnosis was made in $57 \%$ of cases with chest radiography and $76 \%$ of cases with high resolution computed tomography. High confidence was achieved in $23 \%$ of chest radiograph readings and $49 \%$ of high resolution computed tomography readings; of these readings the correct diagnoses were made in $77 \%$ and $93 \%$ respectively. The implications are that high resolution computed tomography will allow a confident and specific diagnosis of diffuse lung disease to be made considerably more often and more accurately than with chest radiography. As an aside to the main purpose of this study, Mathieson et al showed that HRCT correctly predicted whether transbronchial or open lung biopsy was more appropriate in $87 \%$ of cases, compared with $65 \%$ when chest radiography was used (the criterion being that if sarcoidosis or lymphangitis carcinomatosa was the favoured diagnosis transbronchial biopsy was indicated). This is an important study, which should be repeated in the light of increasing experience with high resolution computed tomography.

Now that the correlations between the high resolution computed tomography appearances and the pathological features of most diffuse lung diseases have been thoroughly studied, other areas receiving attention are functional correlations between the extent of disease as shown by high resolution computed tomography and the results of lung function tests. Longitudinal studies of the rate of change by diffuse lung disease detected by high resolution computed tomography compared with that shown by other monitoring techniques, both imaging and functional, are also needed.

The availability and sophistication of computed tomography machines capable of producing high resolution images continues to increase, but skill in interpreting these images is not increasing at the same rate and remains limited to a few centres. Given the obvious utility of high resolution computed tomography in diffuse lung disease (summarised in the table), chest physicians and radiologists should now consider high resolution computed

Summary of the role of high resolution computed tomography in the assessment of interstitial lung disease

1 Confirming or excluding questionable shadowing on a chest radiograph

2 Investigation of a dyspnoeic patient with abnormal lung function test values and an apparently normal chest radiograph

3 Characterisation of disease-may provide specific diagnosis

4 Disclosing coexisting disease-for example, emphysema

5 Providing a guide to the type and site of lung biopsy tomography a routine and indispensable part of the investigation and assessment of a patient with suspected diffuse lung disease.

1 Epler GR, McCloud TC, Gaensler EA, Mikus JP, Carrington CB. Normal chest roentgenograms in chronic diffuse infiltrative lung disease. $N$ Engl J Med 1978;298:934-9.

$2 \mathrm{McCloud}$ TC. Diffuse infiltrative lung disease. In: Putman $\mathrm{CE}$, ed. Pulmonary diagnosis-imaging and other techniques. New York: Appleton Century Croft, 1981:125-53.

$3 \mathrm{Kreel} \mathrm{L}$. Computed tomography of interstitial pulmonary disease. J Computer Assisted Tomogr 1982;6:181-99.

4 Holt RM. Diffuse lung disease. Current Opinion in Radiology. 1990;2:338-50.

5 Mayo JR, Webb WR, Gould R, et al. High resolution CT of the lungs: an optimal approach. Radiology 1987;163: 507-10.

6 Evans SH, Davis R, Cooke J, Anderson W. A comparison of radiation doses to the breast in computed tomographic chest examinations for two scanning protocols. Clin Radiol 1989;40:45-6.

7 Murata K, Itoh $\mathrm{H}$, Todo G, et al. Centrilobular lesions of the lung: demonstration by high-resolution CT and pathologic correlation. Radiology 1986;161:641-5.

8 Friedman PJ. Pulmonary patterns: the concepts of alveolar and intestitial disease. In: Potchen EJ. Current concepts in radiology. St Louis: Mostry, 64-106.

9 McCloud TC, Carrington CB, Gaensler EA. Diffuse infiltrative lung disease: a new scheme for description. Radiology 1983;149:353-63.

10 Naidich DP, Zerhouni EA, Siegelman SS. Computed tomography of the thorax. New York: Raven Press, 1984:219-33.

11 Stein MG, Mayo J, Müller N, Aberle DR, Webb WR, Gamsu G. Pulmonary lymphangitic spread of carcinoma: appearance on CT scans. Radiology 1987;162:371-5.

12 Hamper UM, Fishman EK, Khouri NF, Johns CJ, Wang $\mathrm{KP}$, Siegelman SS. Typical and atypical manifestations of pulmonary sarcoidosis. J Computer Assisted Tomog 1986;10:928-36.

13 Rockoff SD, Rohatgi PK. Unusual manifestations of thoracic sarcoidosis. AJR 1985;144:513-28.

14 Müller NL, Miller RR, Webb WR, Evans KG, Ostrow DN. Fibrosing alveolitis: CT-pathologic correlation. Radiology 1986;160:585-8.

15 Strickland B, Strickland NH. The value of high definition, narrow section computed tomography in fibrosing alveolitis. Clin Radiol 1988;39:589-94.

16 Harrison KM, Glanville AR, Strickland B, et al. Pulmonary involvement in systemic sclerosis: the detection of early changes by thin section CT scan, bronchoalveolar lavage and $99 \mathrm{~m}$ Tc-DTPA clearance. Respir Med 1989;83: and 99

17 Wiggins J, Strickland B, Turner-Warwick M. Combined cryptogenic fibrosing alveolitis and emphysema: the value of high resolution computed tomography in assessment. Respir Med 1990;84:365-9.

18 Müller NL, Staples CA, Miller RR, Vedal S, Thurlbeck WM, Ostrow DN. Disease activity in idiopathic pulmonary fibrosis: CT and pathologic correlation. Radiology 1987;165:731-4.

19 Hansell DM, Wells AU, du Bois R, Corrin B. Disease activity in fibrosing alveolitis: assessment by high resolution CT with histological correlation. Clin Radiol 1990;42:375.

20 Chinet T, Jaubert F, Dusser D, Danel C, Chrétien J Huchon GJ. Effects of inflammation and fibrosis on pulmonary function in diffuse lung fibrosis. Thorax 1990;45:675-8.

21 Aberle DR, Gamsu G, Ray CS, Feuerstein IM. Asbestosrelated pleural and parenchymal fibrosis: detection with high resolution CT. Radiology 1988;166:729-34.

22 Staples CA, Gamsu G, Ray CS, Webb WR. High resolution computed tomography and lung function in asbestosexposed workers with normal chest radiographs. Am Rev Respir Dis 1989;139:1502-8.

23 Hansell DM, Moskovic E. High resolution computed tomography in extrinsic allergic alveolitis. Clin Radiol 1991;43:8-12.

24 Rimmer MJ, Dixon AK, Flower CDR, Sikora K. Bleomycin lung: computed tomographic observations. $\mathrm{Br} J$ Radiol 1985;58:1041-5.

25 Aberle DR, Hansell DM, Brown K, Tashkin DP. Lymphangiomyomatosis: CT, chest radiographic and functional correlations. Radiology 1990;176:381-7.

26 Müller NL, Chiles C, Kullnig P. Pulmonary lymphangiomyomatosis: correlation of CT with radiographic and myomatosis: correlation of CT with radiograph
functional findings. Radiology 1990;175:335-9.

27 Moore ADA, Godwin JD, Müller NL, et al. Pulmonary Histiocytosis X: comparison of radiographic and $\mathrm{CT}$ findings. Radiology 1989;172:249-54.

28 Lenoir S, Grenier P, Brauner MW, et al. Pulmonary lymphangiomyomatosis and tuberous sclerosis: comparison of radiographic and thin-section CT findings. Radiology 1990;175:329-34.

29 Mathieson JR, Mayo JR, Staples CA, Müller NL. Chronic diffuse lung disease: comparison of diagnostic accuracy of CT and chest radiography. Radiology 1989;171:111-6. 